\title{
Functional analysis of a promoter variant identified in the CFTR gene in cis of a frameshift mutation
}

\begin{abstract}
Victoria Viart $^{1,2}$, Marie Des Georges ${ }^{1,3}$, Mireille Claustres ${ }^{1,2,3}$ and Magali Taulan ${ }^{\star, 1,2}$
In monogenic diseases, the presence of several sequence variations in the same allele may complicate our understanding of genotype-phenotype relationships. We described new alterations identified in a cystic fibrosis (CF) patient harboring a $48 C>\mathbf{G}$ promoter sequence variation associated in cis of a 3532AC $>$ GTA mutation and in trans with the F508del mutation. Functional analyses including in vitro experiments confirmed the deleterious effect of the 3532GTA frameshift mutation through the creation of a premature termination codon. The analyses also revealed that the $48 \mathrm{G}$ promoter variant has a negative effect on both transcription and mRNA level, thus demonstrating the importance of analyzing all mutations or sequence variations with potential impact on CF transmembrane conductance regulator processing, even when the two known disease-causing mutations have already been detected. Our results emphasize the need to perform, wherever possible, functional studies that may greatly assist the interpretation of the disease-causing potential of rare mutation-associated sequence variations.
\end{abstract}

European Journal of Human Genetics (2012) 20, 180-184; doi:10.1038/ejhg.2011.161; published online 17 August 2011

Keywords: CFTR; promoter sequence variation; frameshift mutation; functional analysis

\section{INTRODUCTION}

Cystic fibrosis (CF, MIM\# 219700) is the most common lethal autosomal recessive disease affecting the Caucasian population, with an average prevalence of 1 in 3000 births. Birth prevalence varies from country to country and with ethnic background. ${ }^{1}$ Although in its classical form, CF affects the physiology of several organs including the pancreas and the gastrointestinal and reproductive tracts, the morbidity is mainly due to pulmonary defects. ${ }^{2}$ It is caused by mutations in the CF transmembrane conductance regulator (CFTR; MIM\# 602421) gene, for which over 1850 sequence variations have been reported to date (http://www.genet.sickkids.on.ca/cftr/). The pathogenicity of several types of mutations such as nonsense, frameshift and splicing mutations are obvious, whereas the interpretation of the molecular consequences of others, such as silent mutations $s^{3,4}$ or those identified in non-coding regions, ${ }^{5,6}$ is arduous. In addition, the CFTR gene is characterized by the presence of a number of complex alleles, that is, at least two mutations in cis on the same chromosome that generally hinder the establishment of genotype-phenotype correlations. Mutations found in cis can modulate the effect of the principle mutation. The mutation $\mathrm{R} 117 \mathrm{H}$ occurring in cis with the 5-thymidine (5T) tract variant in intron 8 generally results in pancreatic sufficient CF and as such, is considered as a mild mutation, whereas $\mathrm{R} 117 \mathrm{H}$ in cis with the $7 \mathrm{~T}$ is mainly considered as a CFTR-related disease-associated mutation with low penetrance. ${ }^{7}$ Complex alleles may also involve alterations in the CFTR promoter region, as is the case for the $(-102 \mathrm{~T}>\mathrm{A} ; \mathrm{S} 549 \mathrm{R})+(\mathrm{F} 508 \mathrm{del})$ genotype for which the promoter sequence variation is associated with an increase in CFTR expression and a moderate clinical phenotype. ${ }^{8}$

Here we report the functional analysis of a promoter variant associated in cis with a frameshift mutation $(48 \mathrm{C}>\mathrm{G} ; 3532 \mathrm{AC}>$
GTA)+(F508del) identified in a patient with a classic form of CF as characterized by a positive sweat test, pulmonary symptoms, digestive manifestations and pancreatic insufficiency. The two alterations $48 \mathrm{C}>\mathrm{G}$ and $3532 \mathrm{AC}>\mathrm{GTA}$ are rare, as they have been identified in only one patient and none have been found to occur alone elsewhere. In our continuing efforts to assist in the interpretation of uncharacterized mutations, we assessed the contribution of each alteration towards the disease phenotype. Although the molecular defect induced by the 3532AC $>$ GTA mutation (frameshift mutation) seems clear, we also evaluated whether the $48 \mathrm{C}>\mathrm{G}$ promoter sequence variation modulates the effect of the 3532AC $>$ GTA mutation.

\section{MATERIALS AND METHODS}

\section{Nomenclature of mutations}

For convenience to readers, we used legacy nomenclature as previously reported to the International Consortium Mutation Database (http://www.genet.sickkids. on.ca/cftr/). According to the Human Genome Variation Society nomenclature, the two described alterations $48 \mathrm{C}>\mathrm{G}$ and $3532 \mathrm{AC}>\mathrm{GTA}$ correspond to c. $-85 \mathrm{C}>\mathrm{G}$ and c.3400_3401delACinsGTA, respectively (GenBank NM_000492.3), where nucleotide number 1 corresponds to the A of the ATG translation initiation codon (previously the A of the ATG was numbered 133).

\section{Plasmid constructs}

To study the impact of the mutations, several CFTR constructions were created by direct mutagenesis (QuickChange II site directed mutagenesis kit, Agilent, Massy, France). The $48 \mathrm{C}>\mathrm{G}$ sequence variation was introduced either in the CFTR promoter upstream of the Luciferase cDNA (pGL3b vector) or in the CFTR promoter upstream of the wild-type (WT) CFTR cDNA (pcDNA3-1 vector). The $3532 \mathrm{AC}>\mathrm{GTA}$ was introduced in the CFTR cDNA under the control of either the CFTR promoter (WT or altered) or the CMV promoter in the pcDNA3-1 vector. The 3532AC > GTA was also introduced in a pSPL3hybrid minigene containing WT CFTR exon 18 and flanking introns ( $150 \mathrm{bp})$.

${ }^{1}$ INSERM U827, Laboratoire de Génétique de Maladies Rares, Montpellier, France; ${ }^{2}$ Université Montpellier I, UFR de Médecine, Montpellier, France; ${ }^{3} \mathrm{CHU}$ Montpellier, Hôpital Arnaud de Villeneuve, Laboratoire de Génétique Moléculaire, Montpellier, France

${ }^{*}$ Correspondence: Dr M Taulan, INSERM U827, Laboratoire de Génétique de Maladies Rares, Montpellier, France. Tel: +334 11759879 ; Fax: +33 411759882 ; E-mail: magali.taulan@inserm.fr

Received 20 April 2011; revised 29 June 2011; accepted 15 July 2011; published online 17 August 2011 
All constructs were verified by sequencing. All the oligonucleotides used for generating the constructs are available on demand.

\section{Transfection assays}

The A549 pulmonary epithelial cell line was grown in DMEM/F12, and the Cos7 simian fibroblast cell line in DMEM, at $37^{\circ} \mathrm{C}$ under $5 \% \mathrm{CO}_{2}$. Each medium was completed with 5\% SVF (Eurobio, Courtaboeuf, France), $1 \%$ Ultroser G (Pall, Saint Germain en Laye, France), 1\% antibiotics (Invitrogen, Villebon sur Yvette, France) and 1\% L-glutamine (Invitrogen). All transient transfections were realized with Polyfect (Qiagen, Courtaboeuf, France) in 6-well plates (BD falcon, Le Pont de Claix, France), with $1500 \mathrm{ng}$ of indicated vector. For the reporter assay, cells were transfected in 96-well plates (BD falcon) using Fugene6 (Roche Applied Science, Meylan, France), with $60 \mathrm{ng}$ of indicated reporter vector and 6 ng of pRL-SV40 (Renilla Luciferase) to normalize for transfection efficiency. Samples were harvested $48 \mathrm{~h}$ after transfection. A stable A549 cell line, containing WT or 48G-altered CFTR promoter was generated as previously described. ${ }^{6}$

\section{Protein truncation test}

In the first step, the CFTR fragment of interest was amplified from CFTR cDNA, using specific primers with the addition of the T7 promoter at the $5^{\prime}$-end. ${ }^{9}$ To generate WT and mutated peptides encompassing the 3532 position labeled with ${ }^{35} \mathrm{~S}$-methionine, we used the TNT T7 Quick Coupled Transcription/Translation System (Promega, Charbonnieres, France). Produced peptides were then separated by migration on a $12 \%$ denaturing acrylamide gel. After fixation and amplification, the signal was exposed on a Maximum Resolution film (Kodak, VWR, Pessac, France) during $12 \mathrm{~h}$. Molecular weights were calculated with compute $\mathrm{pl} / \mathrm{Mw}$ in ExPASy tools (Expasy Bioinformatics Resource portal, 'Compute pl/Mw tool', http://web.expasy.org/compute_pi/).

\section{Western blot}

Whole protein extracts were directly collected in Laemmli buffer and sonicated before their loading on a 7\% SDS-polyacrylamide gel. After migration, proteins were transferred onto a PVDF membrane (Westran Clear Signal, Whatman, Dominique Dutscher, Issy les Moulineaux, France), which was blocked with 5\% skimmed milk in PBS-Tween for $1 \mathrm{~h}$. Membranes were incubated overnight with anti-CFTR primary antibody (1:400, clone MM13-4 recognizing an N-terminus epitope, Millipore, Molsheim, France) or anti-Lamin A/C primary antibody (1:10000, Upstate, Millipore). After $1 \mathrm{~h}$ of incubation with the anti-mouse secondary antibody, proteins were revealed with Immobilon Western Chemiluminescent HRP Substrate (Millipore) on a chemiluminescence detection film (Kodak).

\section{Quantification of transcript level}

Total mRNAs were purified with RNeasy plus Mini Kit (Qiagen) as recommended by the manufacturer. Genomic DNA was removed with DNase I amplification grade (Invitrogen). After treatment with RNasin (Promega), RT-PCR was performed on $1 \mu \mathrm{g}$ of RNA with random primers and MML-V reverse transcriptase (Invitrogen). Quantitative PCR was realized with 1:50 diluted cDNA and amplified with the LightCycler 480 SYBR Green I Master (Roche Applied Science). CFTR cDNA was quantified and normalized by the quantification of GAPDH and $\beta$-actin housekeeping genes. Controls with no template or reverse transcriptase were also included. All PCR reactions were performed in triplicate in at least three independent experiments.

\section{Minigene assay}

Total mRNA extraction and RT-PCR were performed as described above for the quantification of transcript level. PCR was then performed using specific primers of pSPL3 plasmid and PCR Master Mix (Promega). Fragments were separated on a $1.5 \%$ agarose gel.

\section{Reporter assay}

The experiment was performed as previously described. ${ }^{6}$ Firefly and Renilla Luciferase activities were measured $48 \mathrm{~h}$ after transfection by the DualLuciferase Reporter Assay System (Promega) according to the manufacturer's recommendations. Luciferase activities are representative of at least three independent experiments, with each construct tested in triplicate per experiment.

\section{Quantitative chromatin immunoprecipitation assays (Q-ChIP)}

Q-ChIP was performed as previously described, except for minor modifications. ${ }^{6}$ Purified crosslinked chromatin was immunoprecipitated using $3 \mu \mathrm{g}$ of anti-E2F, anti-MZF1 (myeloid zinc finger 1) or anti-Sp1 (specific protein 1) antibodies. As a control for non-specific binding of DNA, $3 \mu \mathrm{g}$ of anti-HA, anti-goat or anti-mouse antibodies were used. All antibodies were obtained from Santa Cruz, Clinisciences (Montrouge, France), except for anti-HA, which was bought from Roche Applied Science. Input and immunoprecipitated DNAs were analyzed after their amplification on the LightCycler 480 real-time PCR system using the LightCycler 480 Probes Master, specific primers for the CFTR minimal promoter region encompassing the $48 \mathrm{C}>\mathrm{G}$ sequence variation and probe \#62 (Roche Applied Science). All PCR reactions were performed in triplicate. Experiments were performed at least three times and expressed relative to the input signal and to non-specific immunoprecipitated chromatin.

\section{Statistical analyses}

Data are expressed as the mean \pm SE. Paired comparisons were made using Student's $t$-test with InStat (GraphPad Software, version 3.0, Instat 3 folder, San Diego, CA, USA). Data were considered statistically significant at $P<0.05$ and extremely significant at $P<0.0001$.

\section{RESULTS}

Confirmation of the deleterious effect of the 3532AC $>$ GTA frameshift mutation

We firstly hypothesized that the probable change in the open reading frame induced by the 3532GTA frameshift mutation generates a premature termination codon (PTC; Figure 1a). We therefore performed a protein truncation test (PTT) by transcribing and translating in vitro the WT and mutated CFTR fragments. Results of electrophoresis showed a shift in the molecular weight of the synthesized peptides between CFTR-WT and CFTR-3532GTA conditions (Figure $1 \mathrm{~b}$ ). The presence of a band with the expected molecular weight confirmed the creation of a PTC, 21 codons after the mutation, at the end of the last transmembrane segment.

To investigate further the several additional bands in the CFTR3532GTA compared with the WT conditions revealed by our PTT, we assessed the effect of the 3532GTA mutation on CFTR maturation by western blot. Total proteins from cells transfected with an expression vector containing WT or mutated CFTR cDNA, downstream of the CMV promoter, were used for immunoblot assay. Total proteins from cells transfected with the construction containing WT CFTR cDNA resulted in typical bands including band $\mathrm{C}$ (mature, fully glycosylated protein, $170 \mathrm{kDa}$ ) and band $\mathrm{B}$ (immature, core-glycosylated protein, $140 \mathrm{kDa}$; Figure 1c). As a control, we used the F508del mutation, known to induce protein misconformation and in turn, its premature degradation. No bands were detected, however, for the 3532GTA mutation as in the non-transfected condition, whereas protein was present, as confirmed using anti-Lamin A/C primary antibody. The mutated sequence therefore prevents protein synthesis, suggesting that transcripts bearing this PTC are potential targets for degradation through nonsense-mediated mRNA decay (NMD) and/or for exon skipping through nonsense-associated alternative splicing (NAS).

We next assessed the impact of the 3532GTA allele on the mRNA level or splicing process. Evaluation of mRNA amounts revealed that the 3532GTA mutation induces a decrease in CFTR transcript levels of more than $80 \%$, compared with the WT condition in Cos-7 cells (Figure 1d). The same results were obtained in A549 lung-specific cells (data not shown). Because this frameshift mutation might also produce both correctly and aberrantly spliced mRNA, we used a 
a

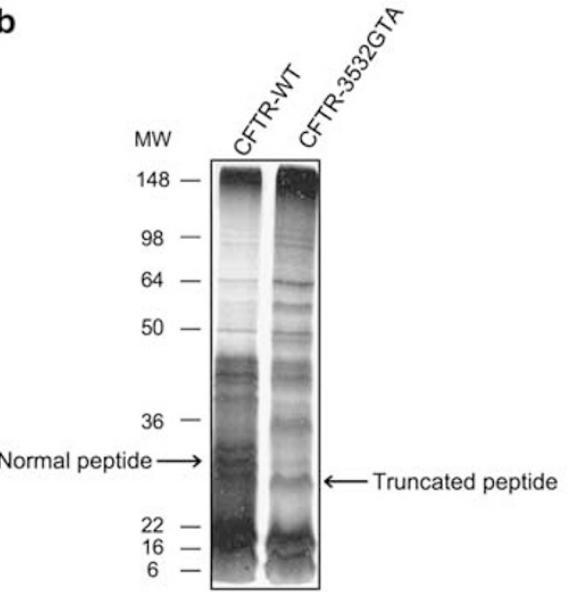

C

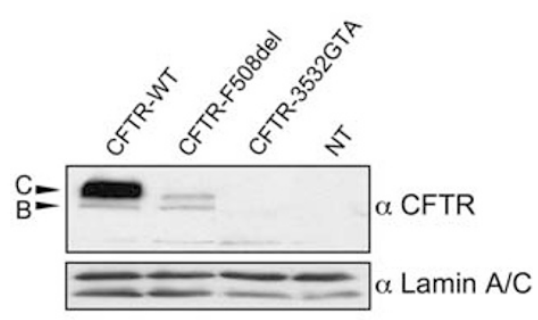

d

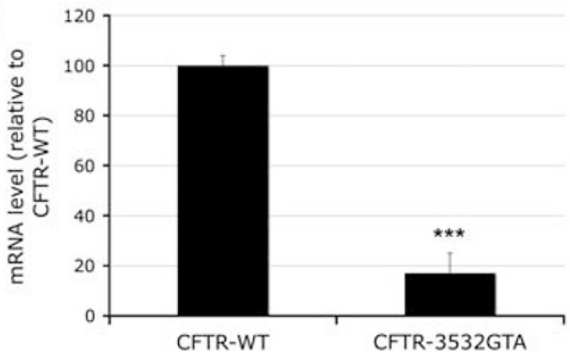

e

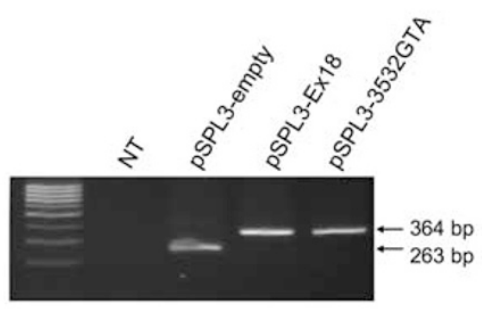

Figure 1 Confirmation of the deleterious effect of the 3532AC>GTA frameshift mutation. (a) Schematic representation of the location of the frameshift mutation, premature stop codon and anti-CFTR primary antibody. The star represents the location of the frameshift mutation in exon 18, coding for the last transmembrane region of MSD2, STOP in the square shows the position of the PTC. (b) Analysis of the impact of the 3532GTA mutation by PTT. Confirmation of the creation of a PTC in the mutant context (CFTR-3532GTA) compared with wild type (CFTR-WT). The presence of the normal or truncated peptide was defined by expected molecular weight. (c) Analysis of the impact of the 3532GTA mutation on protein maturation by western blot assay. Proteins were extracted from Cos-7 cells non transfected (NT) or transfected with either WT (CFTR-WT) or mutated (CFTR-F508del or CFTR-3532GTA) constructs. Immunoblots were performed using either anti-CFTR or anti-Lamin A/C antibodies. (d) Assessment of the frameshift mutation at the CFTR mRNA level. mRNA was extracted from Cos-7 cells transfected with wild-type (CFTR-WT) or mutated (CFTR-3532GTA) constructs and analyzed by RT-quantitative PCR. The data are normalized to the GAPDH and $\beta$-actin transcript level. (e) Analysis of the effect of 3532GTA on splicing by hybrid minigene. Total mRNAs were extracted from Cos-7 cells, transfected with either empty vector (pSPL3-empty) or constructs with exon 18, containing or not containing the 3532GTA mutation. Values are extremely significant at $* * * P<0.0001$ when compared with the WT context.

minigene assay to investigate whether the second control mechanism NAS could account for the observed inhibited synthesis. Hybrid minigene assay showed no difference between WT and mutated contexts (Figure 1e). As alternative splicing events have been proposed to differ between cell lines, ${ }^{10}$ we verified that the same results were obtained in the other tested cell line A549 (data not shown). These results indicate that degradation of the transcript bearing the PTC in exon 18 is the result of NMD rather than NAS.

\section{Characterization of the pathogenicity of the $48 C>G$ promoter sequence variation}

To evaluate the effect of the $48 \mathrm{G}$ variant on transcriptional regulation, we performed a reporter assay. Luciferase activity, reflecting CFTR promoter control, indicated a decrease of about $40 \%$ in the $48 \mathrm{G}$ variant context compared with WT (Figure 2a). We next assessed differences in CFTR mRNA amounts and found that the $48 \mathrm{G}$ allele induces a decrease of $75 \%$ in the CFTR transcript level when compared with the WT (Figure 2b). These results suggest that the $48 \mathrm{G}$ promoter variant, in addition to its deleterious effect on transcription, may also disrupt the stability of the CFTR transcript. When associated in cis of the 3532GTA frameshift mutation, the $48 \mathrm{G}$ variant did not, however, increase the impact of 3532GTA alone on the mRNA level. Finally, we investigated which regulatory motifs, encompassing the $48 \mathrm{C}>\mathrm{G}$ promoter sequence variation, were involved. Bioinformatics analyses predicted a change in binding capability of E2F, MZF1 and Sp1 transcription factors. We therefore performed Q-ChIP on WT or sequence-varied promoters, using specific anti-E2F, anti-MZF1 and anti-Sp1 antibodies. Results show that none of the three transcription 
a
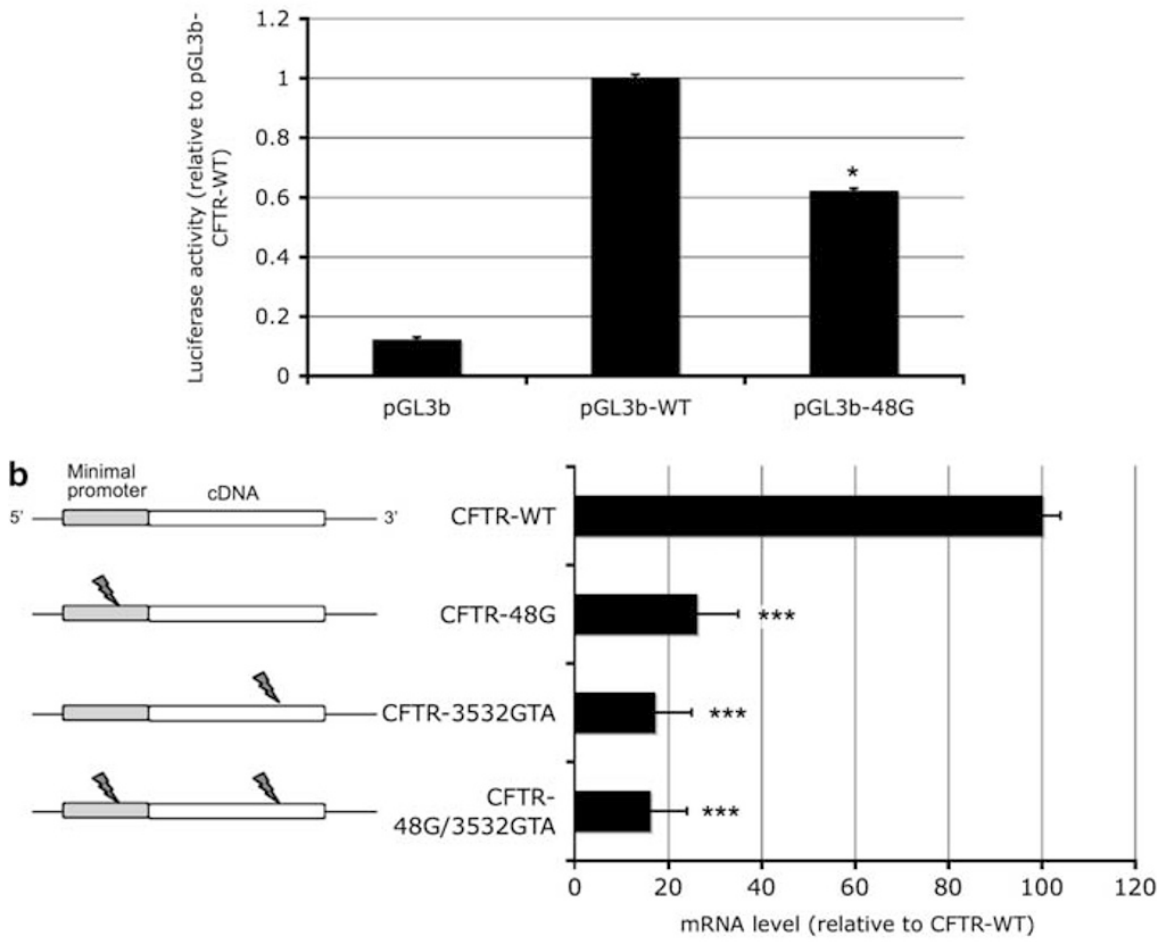

C

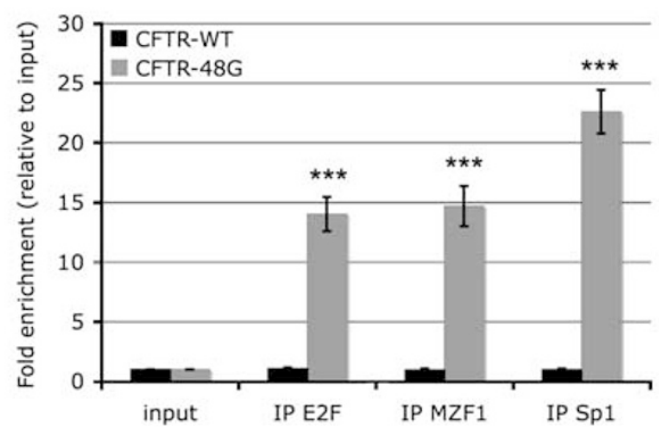

Figure 2 Characterization of the pathogenicity of the $48 C>G$ promoter sequence variation. (a) Impact of the $48 \mathrm{G}$ variant at the transcriptional level by reporter assay. Luciferase was measured in cell lines derived from human lung (A549 cells) and transfected with empty plasmid or with the constructs containing either the 48C WT allele (CFTR-WT) or the 48G sequence varied allele (CFTR-48G). The Firefly Luciferase activity was normalized to Renilla Luciferase. (b) Impact of the alterations at the CFTR mRNA level. mRNA was extracted from A549 cells transfected with WT (CFTR-WT) or altered constructs, either 48G alone (CFTR-48G) or in combination with the 3532GTA mutation (CFTR-48G/3532GTA), and then analyzed by RT-quantitative PCR. The data are normalized to GAPDH and $\beta$-actin transcript level. (c) Binding affinity of E2F, MZF1 and Sp1 transcription factors. Chromatin from A549 cells, that stably express constructs containing either wild type (CFTR-WT) or sequence varied (CFTR-48G) promoter, was used for ChIP assay. Chromatin was immunoprecipitated (IP) with specific E2F, MZF1 and Sp1 antibodies. The data are presented relative to non-IP chromatin (input). The results are also normalized to chromatin IP by non-specific antibodies. Values are significant at ${ }^{*} P<0.05$ and extremely significant at ${ }^{* * *} P<0.0001$, compared with the WT context.

factors bound in vivo to the tested region of the WT promoter (Figure 2c), whereas E2F, MZF1 and Spl proteins bound to the sequence-varied promoter with high affinity $(14,15$ and 23-fold enrichment relative to input, respectively), suggesting their putative involvement in the $48 \mathrm{G}$ promoter variant effect on transcriptional control.

\section{DISCUSSION}

Here we have described the contribution of each alteration $(48 \mathrm{C}>\mathrm{G}$ and 3532AC $>$ GTA) to CFTR function. The creation of a PTC with the 3532GTA frameshift mutation has been confirmed using PTT assay. This mutation was shown to have a deleterious effect at both the mRNA and protein levels with results, suggesting the involvement of
NMD in the recognition and degradation of aberrant transcripts. Although no band C was observed by western blot, indicating $100 \%$ degradation, residual CFTR mRNA was obtained. Previous studies have shown the concomitant nature of the translation and folding of CFTR. ${ }^{11,12}$ Our data suggest the role of an additional quality control mechanism that rapidly directs the elimination of the truncated protein (lacking NBD2) by ER-quality control systems.

We next explored the functional impact of the $48 \mathrm{C}>\mathrm{G}$ sequence variation found in the promoter in cis of the frameshift mutation. Study of the $48 \mathrm{G}$ variant shows its deleterious effect at the transcriptional and mRNA levels. This outcome could be, in part, explained by the higher affinity of the altered sequence for the three transcription factors E2F, MZF1 and Sp1. MZF1 is well known for its role in 
regulating cell proliferation, ${ }^{13}$ but along with $\mathrm{Sp} 1$, it is also involved in regulating the expression of the human MUC1 gene, expressed in several epithelial tissues. ${ }^{14}$ Furthermore, MZF1 has previously been identified as a putative inhibitor of the Cftr gene, through its binding in the -834 to -524 region of the mouse promoter. ${ }^{15}$ Here we have shown that MZF1 only binds to the altered CFTR allele (48G), suggesting its partial role in decreasing promoter activity. E2F transcription factors are largely involved in cell cycle regulation, ${ }^{16,17}$ and along with MZF1, regulate genes that are developmentally regulated during cystogenesis. ${ }^{18}$ Moreover, E2F and Sp1 can control the initiation of transcription in TATA-less promoters ${ }^{19,20}$ through their interaction with other proteins. For instance, E2F could superactivate Sp1dependant transcription in DHFR TATA-less promoter. ${ }^{21}$ Transcription factors, through their binding to regulatory regions, either activate or repress the transcription of their target genes by promoting or inhibiting the recruitment of RNA polymerase II, through TSS use. Indeed, E2F and Sp1 have been shown to interact with several proteins of the general transcription machinery, such as TFIIH and TFIID. ${ }^{22-25}$ These data suggest that the binding of these factors putatively modifies the use of the major TSS essential for CFTR transcription during lung development. ${ }^{26,27}$

The identification of alterations in non-coding regions and the determination of the impact of each allele component have several important consequences for recessive disorders such as CF. They permit a better understanding of the complex genotype-phenotype relationships and the opportunity to offer personalized genetic counseling or adapted therapy. These data reinforce the necessity to screen, wherever possible, for additional alterations even when two allelic mutations known to be disease causing are found, and especially when a discrepancy between phenotype and genotype is observed.

\section{CONFLICT OF INTEREST}

The authors declare no conflict of interest.

\section{ACKNOWLEDGEMENTS}

This work was supported by the French association Vaincre La Mucoviscidose, by $\mathrm{PhD}$ fellowships grants to VV (G0804), the Hospital of Montpellier (Centre Hospitalo-Universitaire) and the Institut National de la Santé et de la Recherche Médicale.

1 O'Sullivan BP, Freedman SD: Cystic fibrosis. Lancet 2009; 373: 1891-1904.

2 Chmiel JF, Davis PB: State of the art: why do the lungs of patients with cystic fibrosis become infected and why can't they clear the infection? Respir Res 2003; 4: 8.

3 Bartoszewski RA, Jablonsky M, Bartoszewska S et al: A synonymous single nucleotide polymorphism in DeltaF508 CFTR alters the secondary structure of the mRNA and the expression of the mutant protein. J Biol Chem 2010; 285: 28741-28748.
4 Pagani F, Raponi M, Baralle FE: Synonymous mutations in CFTR exon 12 affect splicing and are not neutral in evolution. Proc Natl Acad Sci USA 2005; 102: 6368-6372.

5 Taulan M, Lopez E, Guittard C et al: First functional polymorphism in CFTR promoter that results in decreased transcriptional activity and Sp1/USF binding. Biochem Biophys Res Commun 2007; 361: 775-781.

6 Lopez E, Viart V, Guittard C et al: Variants in CFTR untranslated regions are associated with congenital bilateral absence of the vas deferens. J Med Genet 2011; 48: 152-159.

7 Kiesewetter S, Macek Jr M, Davis C et al: A mutation in CFTR produces different phenotypes depending on chromosomal background. Nat Genet 1993; 5: 274-278.

8 Romey MC, Guittard C, Carles S, Demaille J, Claustres M, Ramsay M: First putative sequence alterations in the minimal CFTR promoter region. J Med Genet 1999; 36: 263-264.

9 Romey MC, Tuffery S, Desgeorges M, Bienvenu T, Demaille J, Claustres M: Transcript analysis of CFTR frameshift mutations in lymphocytes using the reverse transcriptionpolymerase chain reaction technique and the protein truncation test. Hum Genet 1996; 98: 328-332.

10 Disset A, Michot C, Harris A, Buratti E, Claustres M, Tuffery-Giraud S: A T3 allele in the CFTR gene exacerbates exon 9 skipping in vas deferens and epididymal cell lines and is associated with Congenital Bilateral Absence of Vas Deferens (CBAVD). Hum Mutat 2005; 25: 72-81.

11 Du K, Sharma M, Lukacs GL: The DeltaF508 cystic fibrosis mutation impairs domaindomain interactions and arrests post-translational folding of CFTR. Nat Struct Mol Biol 2005; 12: 17-25.

12 Kleizen B, van Vlijmen T, de Jonge HR, Braakman I: Folding of CFTR is predominantly cotranslational. Mol Cell 2005; 20: 277-287.

13 Gaboli M, Kotsi PA, Gurrieri C et al: Mzf1 controls cell proliferation and tumorigenesis. Genes Dev 2001; 15: 1625-1630.

14 Shiraga T, Winpenny JP, Carter EJ, McCarthy VA, Hollingsworth MA, Harris A: MZF-1 and $\mathrm{DbpA}$ interact with DNase I hypersensitive sites that correlate with expression of the human MUC1 mucin gene. Exp Cell Res 2005; 308: 41-52.

15 Ulatowski LM, Whitmore KL, Romigh T, VanderWyden AS, Satinover SM, Drumm ML: Strain-specific variants of the mouse Cftr promoter region reveal transcriptional regulatory elements. Hum Mol Genet 2004; 13: 1933-1941.

16 van den Heuvel S, Dyson NJ: Conserved functions of the pRB and E2F families. Nat Rev Mol Cell Biol 2008; 9: 713-724.

17 Chen HZ, Tsai SY, Leone G: Emerging roles of E2Fs in cancer: an exit from cell cycle control. Nat Rev Cancer 2009; 9: 785-797.

18 Lantinga-van Leeuwen IS, Leonhard WN, Dauwerse $\mathrm{H}$ et al: Common regulatory elements in the polycystic kidney disease 1 and 2 promoter regions. Eur J Hum Genet 2005; 13: 649-659.

19 Azizkhan JC, Jensen DE, Pierce AJ, Wade M: Transcription from TATA-less promoters. dihydrofolate reductase as a model. Crit Rev Eukaryot Gene Expr 1993; 3: 229-254.

$20 \mathrm{Li} \mathrm{H}$, Liu H, Wang $\mathrm{Z}$ et al: The role of transcription factors Sp1 and YY1 in proximal promoter region in initiation of transcription of the mu opioid receptor gene in human lymphocytes. J Cell Biochem 2008; 104: 237-250.

21 Lin SY, Black AR, Kostic D, Pajovic S, Hoover CN, Azizkhan JC: Cell cycle-regulated association of E2F1 and $\mathrm{Sp} 1$ is related to their functional interaction. Mol Cell Biol 1996; 16: 1668-1675.

22 Gill G, Pascal E, Tseng ZH, Tjian R: A glutamine-rich hydrophobic patch in transcription factor Sp1 contacts the dTAFII110 component of the Drosophila TFIID complex and mediates transcriptional activation. Proc Natl Acad Sci USA 1994; 91: 192-196.

23 Pearson A, Greenblatt J: Modular organization of the E2F1 activation domain and its interaction with general transcription factors TBP and TFIIH. Oncogene 1997; 15: 2643-2658.

24 Smale ST: Core promoters: active contributors to combinatorial gene regulation. Genes Dev 2001; 15: 2503-2508.

25 Liu WL, Coleman RA, Ma E et al: Structures of three distinct activator-TFIID complexes. Genes Dev 2009; 23: 1510-1521.

26 Riordan JR, Rommens JM, Kerem B et al: Identification of the cystic fibrosis gene: cloning and characterization of complementary DNA. Science 1989; 245: 1066-1073.

27 White NL, Higgins CF, Trezise AE: Tissue-specific in vivo transcription start sites of the human and murine cystic fibrosis genes. Hum Mol Genet 1998; 7: 363-369. 